\title{
Encapsulation of nystatin in nanoliposomal formulation: characterization, stability study and antifungal activity against Candida albicans
}

\author{
Ebrahim Saadat $^{1}$, Rassoul Dinarvand ${ }^{1,2}$, Pedram Ebrahimnejad ${ }^{3,4^{*}}$ \\ ${ }^{1}$ Novel Drug Delivery Systems Lab, Faculty of Pharmacy, Tehran University of Medical Sciences, Tehran, Iran \\ ${ }^{2}$ Medical Nanotechnology Research Centre, Tehran University of Medical Sciences, Tehran, Iran \\ ${ }^{3}$ Department of Pharmaceutics, Faculty of Pharmacy, Mazandaran University of Medical Sciences, Sari, Iran \\ ${ }^{4}$ Pharmaceutical Sciences Research Center, Mazandaran University of Medical Sciences, Sari, Iran
}

\begin{abstract}
In this study, nystatin liposomal formulation was prepared and characterized. The physicochemical properties of formulations including vesicle size, drug entrapment stability and in vitro release were studied. The highest entrapment efficiency of nystatin into liposomes was obtained about $70 \%$ when cholesterol (CHO) was added to the formulations prepared with dipalmitoylphosphatidylcholine. In addition, the drug entrapment efficiency was decreased when distearoylphosphatidylcholine was used but it was improved by addition of $\mathrm{CHO}$ and hydration with $9 \%$ sucrose solution. Liposomes with uniform size distribution and average size of $100 \mathrm{~nm}$ were produced. Long term stability study indicated that the lyophilized liposomal nystatin was physically stable for at least 6 months at $4{ }^{\circ} \mathrm{C}$. In vitro anti-fungal activity of liposomal nystatin was found to be more effective than free nystatin against Candida albicans.
\end{abstract}

Keywords: Liposome, lyophilization, antifungal activity, nystatin, nanotechnology

Pharm Biomed Res 2016; 2(1): 44-54

DOI: 10.18869/acadpub.pbr.2.1.44

\section{Introduction}

Polyene antibiotics are a group of macrolide lactones that have antifungal activity against a wide variety of fungi. Administration of this group of antibiotics have recently increased due to the use of immunosuppressive drugs and increasing incidence of AIDS $(1,2)$. Nystatin, the first polyene drug to be identified, is active against a wide variety of fungal pathogens including Candida, Aspergillus, Histoplasma and Coccidioides spp. $(4,5,6)$.
Nystatin has a similar structure to amphotericin B, but has a broader spectrum of action than amphotericin $B$ and is used for the treatment of cutaneous, vaginal and oral candidiases by oral, pleural inhalation and topical administration (7-9). Nystatin, has a broad spectrum of activity, but due to its low solubility in injectable solvents and toxicity, its use in the treatment of systemic fungal infections is limited $(7,8)$. Unfortunately its clinical use, other than by topical application, has been 
shown systemic side effects and toxicity, manifested by thrombophlebitis, fever and rigors (10). In addition it is regarded as toxic when given intravenously, but it is not absorbed across intact skin or mucous membranes (10).

Liposomes have previously been used as carriers for various of hydrophobic, poorly soluble drugs, and they have been shown to reduce side effects and ameliorate toxicity of certain drugs $(11,12)$. Liposomes can also provide slow release of an encapsulated drug, resulting in sustained exposure to disease parts and enhanced efficacy (13-15). As formulation of nystatin in concentrated pharmaceutical delivery systems for parenteral administration is very difficult, one way of improving the solubility of certain drugs is to formulate them into liposomes (16). In the case of nystatin, besides these advantages, the properties of drug, i.e. potential of antifungal activity, make it worthwhile to develop liposomal formulation. Therefore based on mentioned results, a liposome-based formulation was developed in this study. The schematic structures of formulation ingredients including nystatin, dipalmitoylphosphatidylcholine (DPPC) and distearoylphosphatidylcholine (DSPC) are also shown in Figure 1. Due to strong rational for preparation of nystatin liposomal formulation we attempted to optimize variables for obtaining liposomes with the best possible drug entrapment and stability. Also drug release kinetic and anti-fungal activity of LE-Nystatin and free nystatin were studied.

\section{Materials and methods}

Nystatin (USP 37) was purchased by
Jaber Ebne Hayan pharmaceutical Co. (Iran). Cholestrol (CHOL) were purchased from Sigma-Aldrich (USA) and used without further purification. DSPC and DPPC were obtained from Lipoid $\mathrm{GmbH}$ (Germany). Polycarbonate filters (diameter $19 \mathrm{~mm}$, pore diameter 100 nm) were obtained from Avestin Inc. (Canada). RPMI 1640 medium (with L-glutamine and without bicarbonate) and morpholinopropanesulfonic acid (MOPS) were purchased from Sigma-Aldrich (USA). Deionized water was used throughout the experiments. In vitro release measurement was carried out at $\mathrm{pH}$ 7.4 at $37{ }^{\circ} \mathrm{C}$ in phosphate buffered saline (PBS). All the other chemicals were reagent grade.

\section{Preparation of liposome}

The formulation ingredients are summarized in Table 1. The LENystatin were fabricated by a thinfilm hydration method described by Moribe K 1999 (8). Briefly; nystatin, DSPC or DPPC, and CHO were mixed and dissolved in $10 \mathrm{~mL}$ of mixture of methanol and chloroform $(1: 1, v / v)$. Organic solvents were slowly removed with rotary evaporator (Büchi Rotavapor R-124, Germany) at $40{ }^{\circ} \mathrm{C}$, to deposit a thin film of dried lipid on the inner wall of the flask. The lipid film was then hydrated with $9 \% \quad(w / v)$ sucrose solution or PBS $\mathrm{pH} 7.4$, and stirred for one hour by rotating the flask at about $200 \mathrm{rpm}$ at $40{ }^{\circ} \mathrm{C}$ until the lipid film was completely hydrated and a homogeneous dispersion was formed to obtain the multi-lamellar vesicles (MLVs). Then they were extruded 


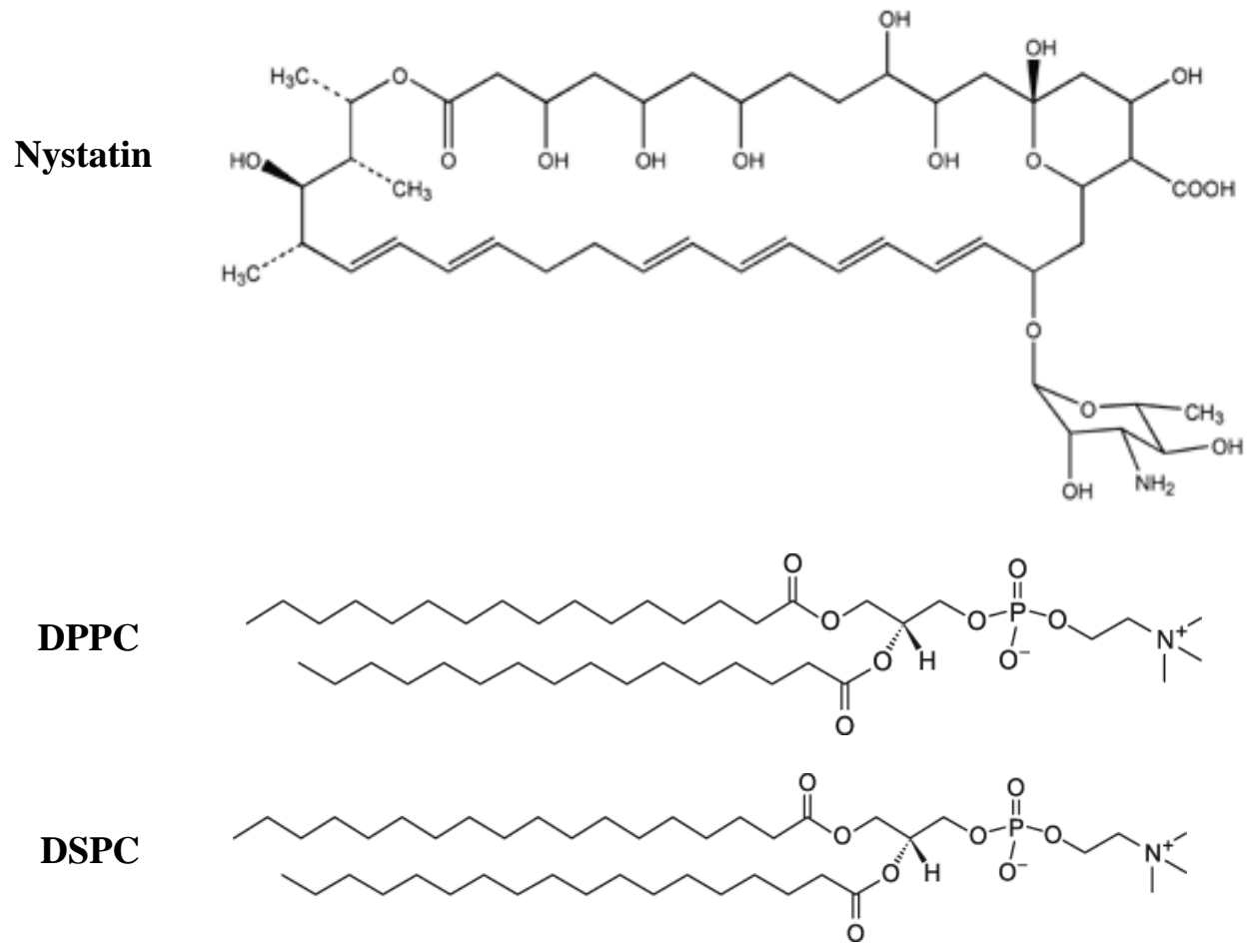

Figure 1 Schematic structure of nystatin, 1,2 -Dipalmitoyl-sn-Glycero-3-

Phosphocholine (DPPC) and 1,2-Distearoyl-sn-Glycero-3-Phosphocholine (DSPC)

Table 1 Various formulations and physiochemical characteristic of LE-Nystatin

\begin{tabular}{ccccccc}
\hline Formulation & Lipid & $\begin{array}{c}\text { Amount of } \\
\text { lipid (mmol) }\end{array}$ & $\begin{array}{c}\text { Amount of } \\
\text { nystatin } \\
(\mathrm{mmol})\end{array}$ & $\begin{array}{c}\text { Amount of } \\
\mathrm{CHO} \\
(\mathrm{mmol})\end{array}$ & Hydrant & $\begin{array}{c}\text { Hydration } \\
\text { time (min) }\end{array}$ \\
\hline $\mathrm{F}_{1}$ & DSPC** & 0.06 & 0.02 & 0.02 & Sucrose 9\% & 30 \\
\hline $\mathrm{F}_{2}$ & DPPC & 0.06 & 0.02 & 0.02 & Sucrose 9\% & 30 \\
\hline $\mathrm{F}_{3}$ & DPPC & 0.06 & 0.02 & 0.02 & Sucrose 9\% & 30 \\
\hline $\mathrm{F}_{4}$ & DPPC & 0.06 & 0.02 & 0.02 & Sucrose 9\% & 30 \\
\hline $\mathrm{F}_{5}$ & DPPC & 0.06 & 0.02 & 0.01 & Sucrose 9\% & 30 \\
\hline $\mathrm{F}_{6}$ & DPPC & 0.06 & 0.02 & 0.03 & Sucrose 9\% & 30 \\
\hline $\mathrm{F}_{7}$ & DPPC & 0.06 & 0.02 & 0.02 & PBS (pH 7.4) & 30 \\
\hline $\mathrm{F}_{8}$ & DPPC & 0.06 & 0.02 & 0.02 & Sucrose 9\% & 15 \\
\hline $\mathrm{F}_{9}$ & DPPC & 0.06 & 0.02 & 0.02 & Sucrose 9\% & 60 \\
\hline
\end{tabular}


through stacked polycarbonate filters of $100 \mathrm{~nm}$ pore size for 11 cycles using a water-jacketed ExtruderTM (Northern lipids, Vancouver BC, Canada) to obtain small unilamellar vesicles (SUVs). Unencapsulated drug was removed from the preparation by dialysis. For doing this process, membranes were soaked overnight in PBS prior to use. The dialysis were maintained at $37{ }^{\circ} \mathrm{C}$ in a shaking water bath and then filled with obtained fornulation. The suspension was then freeze-dried for $48 \mathrm{~h}$ at $-40{ }^{\circ} \mathrm{C}$ (Lyotrap Plus, LTE Scientific Limited, UK) to obtain a fine powder of liposomes, kept in a freezer at $-20{ }^{\circ} \mathrm{C}$ to protect the liposomes from heat and degradation.

\section{Particle size and size distribution}

The particle size and size distribution of the prepared liposomes in the extruded suspension were measured by laser light scattering (Zetasizer Nano ZS, Malvern Instruments Ltd., Malvern, UK). The dried powder samples were suspended in deionised water and sonicated before measurement.

\section{Drug entrapment efficiency $(D E)$}

The concentration of nystatin in liposomes was determined by spectrophotometrically (SCINCO, Seoul, Korea) method at $305 \mathrm{~nm}$. An aliquot of the reconstituted nystatin liposomes was diluted with normal saline and then centrifuged (Sigma $3 \mathrm{k} 30$, Germany) at $21,000 \times \mathrm{g}$ for 30 $\min$ at $4{ }^{\circ} \mathrm{C}$. Then the supernatant was analyzed by the spectrophotometric system. Linear regression analysis data for the calibration plot showed that there was a good linear relationship between response and concentration in the range of 1 to 100 $\mu \mathrm{g} / \mathrm{ml}$; the regression coefficient was 0.9989 and the linear regression equation was $\mathrm{y}=0.0495 \mathrm{x}+0.0234$ ( $\mathrm{n}$ $=3$ ). The absolute calibration curve method was used for calculation. The percentage of the drug entrapped in the liposomes was calculated as follows:

drug entrapment $\%=\frac{\text { total drug }- \text { supernatant drug }}{\text { total drug }} X 100$

\section{In vitro drug release}

In vitro release of nystatin from liposomal formulation was analyzed by membrane dialysis against PBS, $\mathrm{pH} 7.4$ at $37{ }^{\circ} \mathrm{C}$. Briefly, a $2 \mathrm{~mL}$ aliquot of reconstituted LE-Nystatin sample was placed in the membrane dialysis (with a MW cutoff of $10 \mathrm{~K}$ ) and then suspended in a temperaturecontrolled, jacketed flask containing $400 \mathrm{~mL}$ of PBS. At various time intervals, aliquot samples were withdrawn and analyzed by spectrophotometric method described previously. Drug release data was normalized by converting the drug concentration in solution to a percentage of cumulative drug release. The experiments were carried out in triplicate.

In vitro antifungal activity studies

To determine the MIC of the liposomes for the Candida, microdilution method was used. Broth microdilution minimum inhibitory concentrations (MICs) were determined according to National Committee for Clinical Laboratory Standards (NCCLS) recommendations with respect to Candidia albicans ATCC 10261 (22). All tests were repeated thriple. 
The antifungal agents were tested over a final concentration range of 0.125 to $512 \mu \mathrm{g} / \mathrm{ml}$. Test was performed in 96-well round-bottom micro-titer plates. Cell suspensions of Candida spp. were prepared in RPMI 1640 medium with glucose $2 \%$ and buffer phosphate $\mathrm{pH} 7$ adjusted to give a final inoculum concentration of 0.5 McFarland standard $\left(1.5 \times 10^{6} \mathrm{cfu} / \mathrm{ml}\right)$. Final concentration of fungi in individual tubes was adjusted to about $5 \times 10^{3} \mathrm{cfu} / \mathrm{ml}$. The MIC was defined as the lowest concentration at which there was complete inhibition of growth. In the case of liposomes, this amount was calculated according to the percent of drug loading. After $48 \mathrm{~h}$ incubation at $35-37{ }^{\circ} \mathrm{C}$ the plates were examined for possible fungal turbidity and MIC of each test compound was determined as the lowest concentration that could inhibit visible fungal growth. The pharmaceutical preparations were reconstituted according to the manufacturers' instructions. Further dilutions were made with RPMI 1640 medium (with L-glutamine and without bicarbonate), supplemented with glucose (2\%), and buffered to $\mathrm{pH} 7.0$ with $0.165 \mathrm{M}$ MOPS. Nystatin was dissolved in DMF; the solution was diluted with DMF and then with RPMI 1640 medium (22). Control tubes contained no antifungal agent.

\section{Stability study}

The stability of the lyophilized, reconstituted and diluted LE-Nystatin $\left(\mathrm{F}_{2}\right.$ and $\left.\mathrm{F}_{5}\right)$ was evaluated after storage at $-40,4$ and $25{ }^{\circ} \mathrm{C}$ for an extended period of time. The $\mathrm{pH}$, particle size distribution and DE of the samples were determined as a function of the storage time. The drug content in the formulation was determined by analysis method described previously.

\section{Statistical analysis}

One way analyses of variance (ANOVA) test were performed on the data to assess the impact of the formulation variables on the in vitro results. $\mathrm{P}$ values of $<0.05$ were considered significant. The results were analyzed by SPSS-19 software (USA).

\section{Results}

\section{Formulation development}

The data in Table 1 indicated that the best formulation consisted of DPPC/CHO (weight ratio of 50:10 $(w / w)$ which drug to lipid weight ratio is $2: 5(w / w)$.

\section{In vitro drug release}

The in vitro drug release from liposomes $\mathrm{F}_{2}$ and $\mathrm{F}_{5}$ are shown in Figure 2 and Figure 3. In fact, both formulations produced an initial burst release in which drug release was more than $13 \%$ and $18 \%$ for $F_{2}$ and $\mathrm{F}_{5}$, respectively, within the initial sampling time (5 min).

It can be seen that there was approximately $57.1 \% \pm 0.98$ drug release from $\mathrm{F}_{5}$ and $40.2 \% \pm 0.47 \mathrm{drug}$ release from $\mathrm{F}_{2}$ in $\mathrm{PBS}$ buffer over $200 \mathrm{~min}$.

\section{In vitro antifungal activity}

The MIC of free and LE-Nystatin $\left(\mathrm{F}_{2}\right)$ are presented in Table 2. It can be seen that the liposome nystatin possessed antifungal activity against Candidia albicans, ATCC $=10261$. According to the results in this study (Table 2), preparation of LE-Nystatin has 


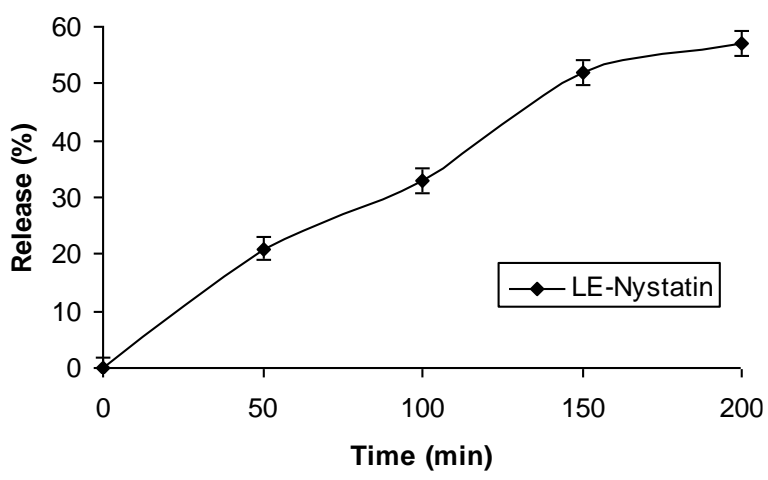

Figure 2 In vitro release curve of nystatin from liposome F5

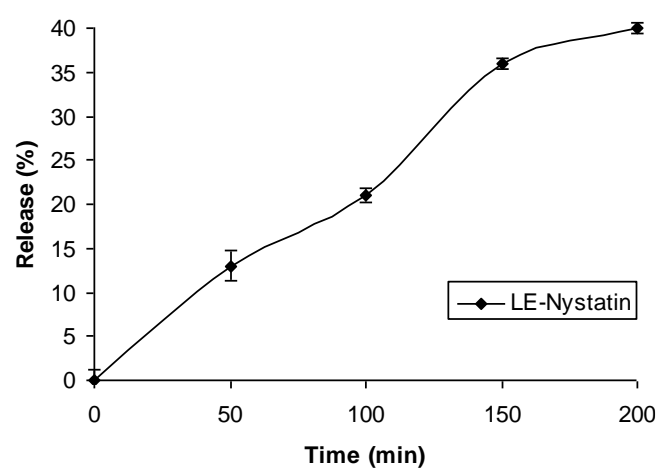

Figure 3 In vitro release curve of nystatin from liposome F2

Table 2 Encapsulation amount of nystatin in various formulations

\begin{tabular}{cc}
\hline Formulation & DE $(\%) \pm$ SD \\
\hline $\mathrm{F}_{1}$ & $45 \pm 0.93$ \\
\hline $\mathrm{F}_{2}$ & $62.5 \pm 0.61$ \\
\hline $\mathrm{F}_{3}$ & $28.7 \pm 0.51$ \\
\hline $\mathrm{F}_{4}$ & $21.9 \pm 0.71$ \\
\hline $\mathrm{F}_{5}$ & $69.1 \pm 0.85$ \\
\hline $\mathrm{F}_{6}$ & $61.7 \pm 0.50$ \\
\hline $\mathrm{F}_{7}$ & $54.8 \pm 0.73$ \\
\hline $\mathrm{F}_{8}$ & $50.3 \pm 0.78$ \\
\hline $\mathrm{F}_{9}$ & $55 \pm 0.86$
\end{tabular}


improved the antifungal efficacy of nystatin on candidia albicans with MIC of $0.5 \mu \mathrm{g} / \mathrm{ml}$ in comparison with free drug with MIC of $2 \mu \mathrm{g} / \mathrm{mL}(p<$ $0.05)$.

\section{Short and long stability of LE-Nystatin}

For indicating the effect of lyophilization on stability of LENystatin, $F_{2}$ and $F_{5}$ (in suspention and lyophilized form) studied at $4{ }^{\circ} \mathrm{C}$ for up to 6 months. The reason for selection of these two formulations with the lowest and highest amount of $\mathrm{CHO}$ was the highest DE among the formulations. It can be obtained in Figure 4 and 5 that significant changes in $\mathrm{DE}$ and $\mathrm{pH}$ of suspension forms were observed in comparison with lyophilized form of liposomes during the course of stability study $(p<0.05)$. Figure 4 illustrates the DE $(\%)$ of lyophilized and eight-fold diluted reconstituted LE-Nystatin $\left(\mathrm{F}_{2}\right)$ at $4{ }^{\circ} \mathrm{C}$ during 6 months. Figure 5 illustrates the $\mathrm{pH}$ of lyophilized and eight-fold diluted reconstituted LE-Nystatin $\left(\mathrm{F}_{2}\right)$ at $4{ }^{\circ} \mathrm{C}$ during 6 months. It was observed that eight-fold diluted reconstituted samples were unstable up to 6 months. This result suggested that the lyophilized liposome showed a more stable state than liposome solution.

Also eight-fold diluted LE-Nystatin physically is stable at $-40{ }^{\circ} \mathrm{C}$ for up to 6 months. No significant changes in mean vesicle size, $\mathrm{pH}$ and $\mathrm{DE}$ were observed during the course of stability study (data not shown).

In addition, eight-fold diluted LENystatin stored at 4 and $25{ }^{\circ} \mathrm{C}$ was physically stable for up to $8 \mathrm{~h}$. At $8 \mathrm{~h}$ time point, the mean vesicle diameter remained relatively constant at both storage temperature and no precipitation or drug crystals were observed (data not shown). Nystatin concentration remained unchanged at both temperature conditions over the course of the stability study (data not shown). Also stability data of $\mathrm{pH}$ for lyophilized and eight-fold diluted reconstituted LE-Nystatin $\left(\mathrm{F}_{2}\right)$ at $4{ }^{\circ} \mathrm{C}$ during 6 months showed in Figure 6.

\section{Discussion}

In the present study, the formulation of liposomes was synthesized in order to elucidate the solubility problems of nystatin, to achieve high drug entrapment in the liposomes, and improve antifungal properties and stability of LE-Nystatin. As shown in Table 1, DE of liposomes prepared using DPPC was higher than those prepared by DSPC. This may be due to the high molecular weight of nystatin and its interaction with the chain and chemical structure of lipid. DPPC with two molecules of palmitoic acid can entrap large amounts of nystatin. Also because of hydrophobic introduction between acyl chain and nystatin, the DE is increased when DPPC phospholipid is used. Besides, the results showed DE depends mainly on the $\mathrm{CHO}$ content. Increasing $\mathrm{CHO}$ from 5 to 10 or 15 mg causes decreasing DE. This difference is significant $(p<0.05)$.

In the absence of any protective agents, vesicle fusion and leakage of internal aqueous contents of liposomes can occur (23). Sugars have been shown to act as protective agents during dehydration/rehydration of liposomes to prevent vesicle fusion and retention of encapsulated compounds within liposomes $(24,25)$. The ability of sugars, such as sucrose, to prevent vesicle fusion was 


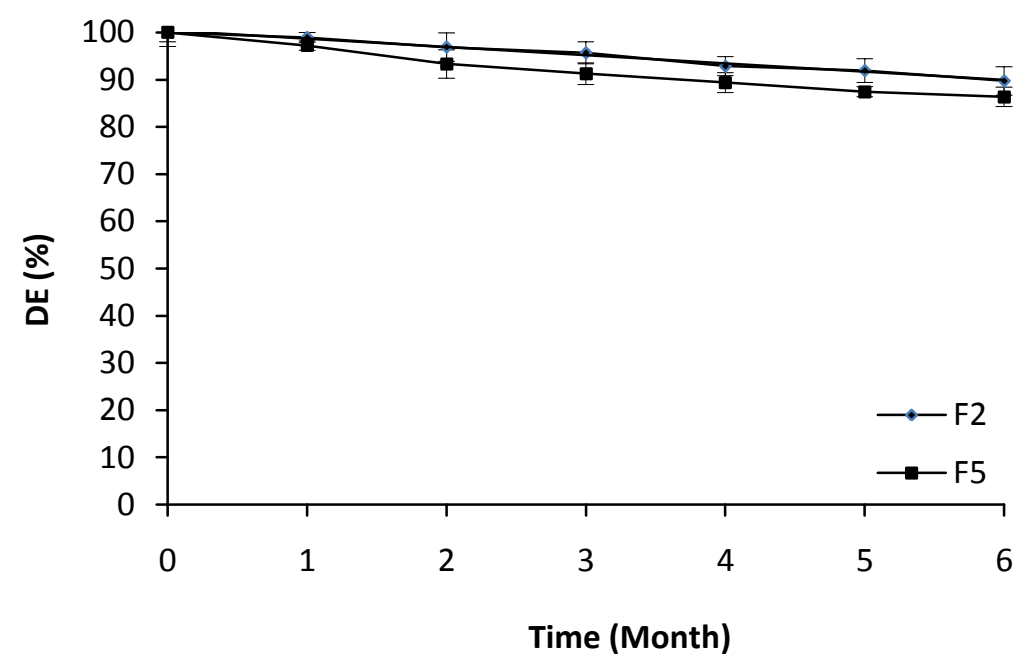

Figure 4 Stability study of lyophilized LE-Nystatin $\left(\mathrm{F}_{2}\right.$ and $\left.\mathrm{F}_{5}\right)$ at $4{ }^{\circ} \mathrm{C}(\mathrm{n}=3)$

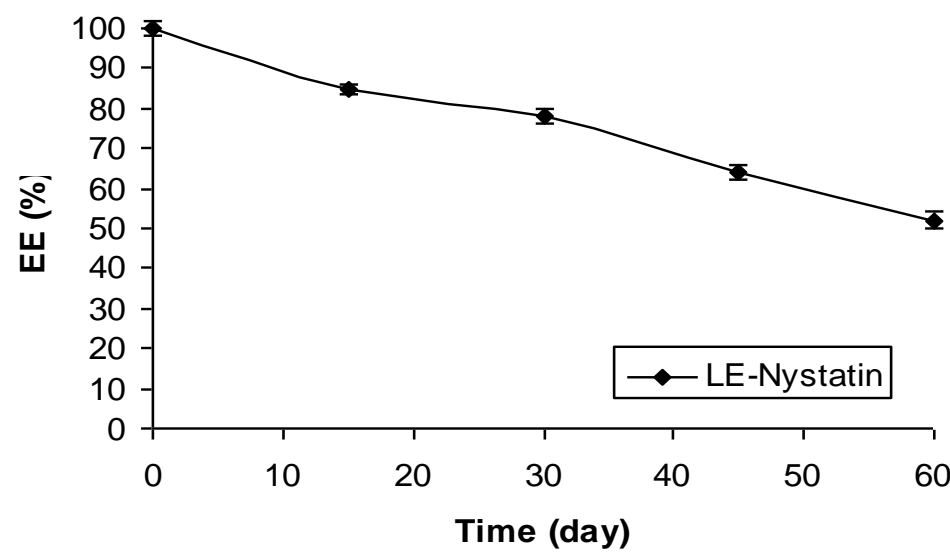

Figure 5 Stability data for eight-fold diluted reconstituted LE-Nystatin $\left(\mathrm{F}_{2}\right)$ at $4{ }^{\circ} \mathrm{C}$

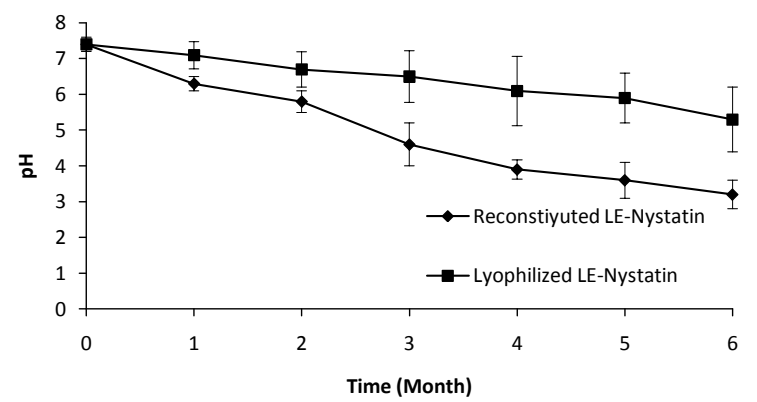

Figure 6 Stability data of $\mathrm{pH}$ for lyophilized and eight-fold diluted reconstituted LE-Nystatin $\left(\mathrm{F}_{2}\right)$ at $4{ }^{\circ} \mathrm{C}$ 
evaluated by measuring the vesicle size and drug entrapment efficiency after the formulation was lyophilized and reconstituted. It was found that sucrose was equally effective in protecting LE-Nystatin during lyophilization and increasing DE (Table 1). These results are correlated with previous studies (26-27). Formulations extruded using $0.1 \mu \mathrm{m}$ membrane filters. Extrusion of liposomes could reduce the size of liposomes about $100 \mathrm{~nm}$ which was confirmed by zeta sizer (data not shown). Sucrose 9\% and buffer phosphate $(\mathrm{pH}$ 7.4) were used as a hydrant. Table 1 shows that sucrose $9 \%$ was a better hydrant when compared with PBS. The optimum time for hydration was $30 \mathrm{~min}$.

LE-Nystatin was characterized with sort of lipid, $\mathrm{CHO}$, and kind of hydrate solution, hydration time and lyophilization effect on DE, drug release and stability of liposomal nystatin.

The initial in vitro burst release of drugs from liposomes has been observed before by other investigators $(2,15)$. The initial burst could be due to the diffusion release of drug distributed at or just beneath the surface of the NPs. A constant slow release of drug in NPs is thought to be due to the involvement of drug molecule entrapped in the polymer matrix which prevents its fast release $(2,15)$. The followed delayed release may be attributed to diffusion of the dissolved drug within the core of the nanoparticle into the dissolution medium (2,15). Overall in vitro release data indicate that liposome based nanoparticles are capable to sustain nystatin release rate successfully.
Moreover, in vitro drug release shows that $\mathrm{CHO}$ reduces the drug release from liposomes suggesting that $\mathrm{CHO}$ and nystatin may have some molecular association reducing drug release. It also suggests that LENystatin would be stable during clinical use when diluted with either normal saline or PBS buffer.

Based on mentioned results in Table 3, LE-Nystatin indicated higher antifungal activity in comparison with free drug. It may be due to the better penetration of liposome into the fungi cells and better delivery of nystatin to its site of action.

Table 3 Minimum inhibitory concentration of LE-Nystatin and free drug liposome against Candidia albicans, $\quad \mathrm{ATCC}=10261$ by microdilution method

\begin{tabular}{cc}
\hline Material & MIC $(\boldsymbol{\mu g} / \mathbf{m l})$ \\
\hline LE-Nystatin & 0.5 \\
\hline Nystatin & 2 \\
\hline Free liposome & - \\
\hline DMF & - \\
\hline
\end{tabular}

Furthermore, similarity structure between liposome and cell membrane of fungi may cause increasing the penetration into fungi cells. Also free liposome and medium as a control group did not have any antifungal effect. This difference is significant ( $p$ $<0.05)$ and correlated with previous studies $(2,4)$.

The stability is always a limitation factor for the application of liposomes. Phospholipids in liposomes are known 
to be sensitive to hydrolysis and oxidation in aqueous medium. Liposomes can be hydrolyzed to form lysophospholipids and free fatty acids. The lysophospholipid can be further hydrolyzed to glycerophosphate compounds and fatty acids. The hydrolytic degradation may change the rigidity of liposomal bilayers, retention of entrapped drug, and alter liposome size and distribution. To enhance the chemical and physical stability of the liposome formulations, lyophilization was used. Lyophilization would be expected to protect the liposome components from hydrolysis as it removes free water from the product, now ever; the preservation of the structural integrity of liposome during dehydration/rehydration process has presented considerable challenges to the pharmaceutical scientists $(26,27)$.

\section{Conclusion}

In conclusion, nystatin liposomes were prepared using DPPC phospholipid in combination with $\mathrm{CHO}$.

\section{References}

1. Pons V, Greenspan D, Lozada NF, McPhail L, Gallant JE, Tunkel A, et al. Oropharyngeal candidiasis in patients with AIDS: randomized comparison of fluconazole versus nystatin oral suspensions. Clin Infect Dis 1997;24: 1204-7.

2. Arikan S, Ostrosky-Zeichner L, LozanoChiu M, Paetznick V, Gordon D, Wallace $\mathrm{T}$, et al. In vitro activity of nystatin compared with those of liposomal nystatin, amphotericin B, and fluconazole against clinical Candida isolates. J Clin Microbiol 2002;40:1406-12.

3. Stanley VC, English MP. Some effects of nystatin on the growth of four Aspergillus
Liposomes were stable with high encapsulation. Drug entrapment was improved when $\mathrm{CHO}$ was combined with phospholipids. The study shows that the antifungal activity of LENystatin was higher than free nystatin. These results may help in the development of effective pharmaceutical formulations for injectable hydrophobic drugs with reducing side effects.

\section{Acknowledgments}

This work was supported by Medical Nanotechnology Research Centre of Ministry of Health of Iran.

\section{Conflict of interest}

The authors declared no potential conflict of interest with respect to the authorship, and/or publication of this study. species. J Gen Microbiol 1965;40:107-18.

4. Elizabeth MJ, Joshua OO, Adrien S, Thomas LW, David W. Comparison of in vitro antifungal activities of free and liposome-encapsulated nystatin with those of four amphotericin B formulations. Antimicrob Agents Chemother 1998;42: 1412-16.

5. Akaike N, Harata N. Nystatin perforated patch recording and its applications to analyses of intracellular mechanisms. Jpn J Physiol 1994:44:433-73.

6. Coutinho A, Prieto M. Cooperative partition model of nystatin interaction with phospholipid vesicles. Biophys J 2003;84: 3061-78. 
7. Schafer KM, Blechschmidt J, Korting HC. Clinical use of oral nystatin in the prevention of systemic candidiasis in patients at particular risk. Mycoses 1996; 39:329-39.

8. Moribe $\mathrm{K}$, Maruyama $\mathrm{K}$, Iwatsuru $\mathrm{M}$. Encapsulation characteristics of nystatin in liposomes: effects of cholesterol and polyethylene glycol derivatives. Int $\mathbf{J}$ Pharm 1999;188:193-202.

9. Newcomer VD, Wright ET, Sternberg TH, Graham JH, Wier RH, Egeberg RO. Evaluation of nystatin in the treatment of coccidioidomycosis in man. Antibiot Annu 1955;3:831-36.

10. Drummond DC, Meyer O, Hong K, Kirpotin DB, Papahadjopoulos D. Optimizing liposomes for delivery of chemotherapeutic agents to solid tumors. Pharmacol Rev1999;51:691-743.

11. Allen TM, Cullis PR. Drug delivery systems: entering the mainstream. Science 2004;303:1818-22.

12. Chen C, Han D, Cai C, Tang X. An overview of liposome lyophilization and its future potential. J Control Release 2010;142:299-311.

13. Sharma A, Sharma US. Liposomes in drug delivery: progress and limitations. Int $\mathbf{J}$ Pharm 1997;154:123-40.

14. Balasubramanian SV, Straubinger RM. Taxol-lipid interactions: taxol dependent effects on the physical properties of model membranes. Biochemistry1994;33:894147.

15. Zhang JA, Xuan T, Parmar M, Ma L, Ugwu S, Ali S, et al. Development and characterization of a novel liposome-based formulation of $\mathrm{SN}-38$. Int $\mathrm{J}$ Pharm 2004;270:93-102.

16. Kunikazu MK, Motoharu I. Spectroscopic investigation of the molecular state of nystatin encapsulated in liposomes. Int $\mathbf{J}$ Pharm 2000;201:37-49.

17. Mehta RT, Hopfer RL, Gunner LA, Juliano RL, Lopez-Berestein G. Formulation, toxicity, and antifungal activity in vitro of liposome-encapsulated nystatin as therapeutic agent for systemic candidiasis. Antimicrob. Agents Chemother 1987;31:1897-900.

18. Wallace TL, Paetznick V, Cossum PA, Lopez-Berenstein G, Rex JH, Anaissie E. Activity of liposomal nystatin against disseminated Aspergillus fumigatus infection in neutropenicmice. Antimicrob Agents Chemother 1997;41:2238-43.

19. Mehta RT, Hopfer RL, McQueen T, Juliano RL, Lopez-Berestein G. Toxicity and therapeutic effects in mice of liposome-encapsulated nystatin for systemic fungal infections. Antimicrob Agents Chemother 1987 31:1901-3

20. Madden TD, Bally MB, Hope MJ, Cullis PR, Schieren HP, Janoff AS. Protection of large unilamellar vesicles by trehalose during dehydration: retention of vesicle contents. Biochim. Biophys. Acta 1985;817:67-74.

21. Dirk H, Petra R, Ann O. Protection of liposomes against fusion during drying by oligosaccharides is not predicted by the calorimetric glass transition temperatures of the dry sugars. Eur Biophys $\mathrm{J}$ 2008;37:503-8.

22. Thomas DM, Marcel BB, Michael JH, Pieter RC, Hugh PS, Andrew SJ. Protection of large unilamellar vesicles by trehalose during dehydration: retention of vesicle contents. Biochim Biophys Acta 1985;817:67-74.

23. Maitani Y, Aso Y, Yamada A, Yoshioka S. Effect of sugars on storage stability of lyophilized liposome/DNA complexes with high transfection efficiency. Int $\mathbf{J}$ Pharm 2008;356:69-75.

24. Molina MC, Allison SD, Anchordoquy TJ. Maintenance of nonviral vector particle size during the freezing step of the lyophilization process is insufficient for preservation of activity: insight from other structural indicators. J Pharm Sci 2001;90:1445-55.

25. Anchordoquy TJ, Carpenter JF, Kroll DJ. Maintenance of transfection rates and physical characterization of lipid/DNA complexes after freeze-drying and rehydration. Arch Biochem Biophys 1997; 348:199-206.

26. Li B, Li S, Tan Y, Stolz DB, Watkins SC, Block LH, Huang L. Lyophilization of cationic lipid-protamine-DNA (LPD) complexes. J Pharm Sci 2000;89:355-64.

27. Molina MC, Armstrong TK, Zhang Y, Patel MM, Lentz YK, Anchordoquy TJ. The stability of lyophilized lipid/DNA complexes during prolonged storage. $\mathbf{J}$ Pharm Sci 2004;93:2259-73. 